\title{
La salud reproductiva de las mujeres inmigrantes: el "plus" de la desigualdad
}

The reproductive health of immigrants women: the "plus" of inequality

María Idoia Ugarte Gurrutxaga

Recibido: 16/09/2019

Aceptado: 11/12/2019

\section{RESUMEN}

La vulnerabilidad de la población inmigrante está ampliamente documentada y se puede comprender teniendo como marco de análisis los determinantes sociales y su implicación en las desigualdades en salud.

En el marco del modelo conceptual de los determinantes sociales de la salud propuesto por la Comisión para Reducir las Desigualdades en Salud en España, el género se postula como uno de los ejes de desigualdad que atraviesan la estructura social y que, en interacción con otros ejes como la clase social, la edad, la etnia o la raza y el territorio, determina jerarquías de poder y conlleva desigualdades en las oportunidades de tener una buena salud.

A pesar del reconocimiento internacional sobre la importancia de la Salud Sexual y Reproductiva (SSR), el ejercicio de los derechos humanos en este campo está fuertemente limitado y existen graves barreras que impiden a las personas - y de forma particular a las mujeres- desarrollar plenamente su sexualidad, así como acceder a servicios y suministros de salud sexual y reproductiva necesarios. Esta situación se agrava en el caso de las mujeres inmigrantes.

Es importante señalar que hay interés y preocupación respecto a la salud obstétrica de la población inmigrante, ya que numerosas investigaciones ponen de manifiesto la existencia de un grave problema de equidad en la salud perinatal de estas mujeres. Su salud reproductiva es mucho peor que la de las mujeres autóctonas.

Entre los factores que contribuyen a la situación de vulnerabilidad de las mujeres inmigrantes destacamos las propias características del contexto migratorio, el abordaje biomédico de la atención a la salud y la escasa competencia cultural del sistema sanitario.

Palabras clave: determinantes sociales de la salud, género, mujeres inmigrantes, salud reproductiva, competencia cultural, feminismo

María Idoia Ugarte Gurrutxaga es profesora en la Escuela de Enfermería y Fisioterapia de la Universidad de Castilla-La Mancha. Correo electrónico: maria.ugarte@uclm.es. ID: https:/ / orcid.org/0000-0003-2413$\underline{3628}$

Cómo citar este artículo: Ugarte Gurrutxaga, María Idoia (2019). La salud reproductiva de las mujeres inmigrantes: el "plus" de la desigualdad. Atlánticas. Revista Internacional de Estudios Feministas, 4 (1), 179196. doi: http: / / dx.doi.org/10.17979/ arief.2019.4.1.3705 


\section{ABSTRACT}

The vulnerability of the immigrant population is widely documented and can be understood by analyzing the social determinants and their implication in health inequalities.

In the framework of the conceptual model of health's social determinants, proposed by the Commission to Reduce Health Inequalities in Spain, gender is postulated as one of the axes of inequality that crosses the social structure and, when it interacts with other axes, such as social class, age, ethnicity or race and territory, determines hierarchies of power and entails inequalities in the chances of good health.

Despite international recognition of the importance of sexual and reproductive health (SRH), in compliance with the human rights in this field is severely limited and there are serious barriers that prevent people - and women in particular - from developing fully their sexuality, as well as having access to necessary sexual and reproductive health services and supplies. This situation is aggravated in the case of immigrant women.

It is important to point out that there is interest and concern regarding the obstetric health of the immigrant population, since many investigations have revealed the existence of a serious problem of equity in these women's perinatal health. Their reproductive health is much worse than indigenous women's.

The incorporation of the intersectional perspective, which arises from the Feminist Theory, in the analysis of this situation facilitates the understanding of the underlying elements in the inequalities in reproductive health of immigrant women.

Among the factors that contribute to the vulnerability of immigrant women, we highlight the characteristics of the migratory context (of differential character between men and women), the biomedical approach to health care and the scarce cultural competence of the health system.

Keywords: feminism, breast cancer, women's health, empowerment. 


\section{INTRODUCCIÓN}

La vulnerabilidad de la población inmigrante está ampliamente documentada y se puede entender teniendo como marco de análisis los determinantes sociales y su implicación en las desigualdades en salud. El género, la clase y la etnia, determinan en gran manera la salud de esta población, situándola en la escala más baja de la posición social. En España, el estudio de las desigualdades en salud ha tenido escasa prioridad política, tal y como se refleja en el documento elaborado por el Ministerio de Sanidad, Servicios Sociales e Igualdad "Avanzando hacia la equidad Propuesta de Políticas e Intervenciones para reducir las desigualdades Sociales en salud en España" (Ministerio de Sanidad, Servicios Sociales e Igualdad, 2015).

Si bien la ejecución de políticas relativas a todos los determinantes sociales es esencial para mejorar la salud y reducir las inequidades, el sector salud tiene un papel vital que desempeñar.

Su conocimiento sobre por qué la salud y la equidad en salud son objetivos compartidos en la sociedad, y en la identificación de la forma en que otros sectores se pueden beneficiar de las medidas relativas a los determinantes sociales, son fundamentales. El sector salud debe colaborar de este modo con otros sectores a fin de reducir las diferencias en la exposición y la vulnerabilidad a las amenazas de salud. De hecho, en lugar de reducir las inequidades en salud, en muchos países del mundo el sector salud suele agudizarlas al ofrecer un mejor acceso y calidad de atención a los segmentos de la sociedad que comparativamente tienen menos necesidades. Así vemos cómo el pago directo de los servicios de salud empuja a 100 millones de personas a la pobreza cada año proteger a los hogares del gasto en salud catastrófico (Xu et al., 2007).

Las políticas sanitarias son un determinante clave en el acceso a los servicios, deberían permitir al sistema sanitario hacer frente al proceso de atención a la población inmigrante de manera adecuada. Sin embargo, actualmente en 
España, el Real Decreto-Ley 16/2012, de 20 de abril, de medidas urgentes para garantizar la sostenibilidad del Sistema Nacional de Salud y mejorar la calidad y seguridad de sus prestaciones la norma legislativa de mayor rango en materia sanitaria, lejos de caminar en este sentido, condiciona el acceso y uso del sistema de salud a determinados requisitos administrativos vinculados a las políticas migratorias. En Castilla-La Mancha, desde el día 1 de marzo de 2016, la Consejería de Sanidad haciendo valer sus competencias en la atención sanitaria, ha ampliado la cobertura facilitando que casi toda la población que reside en esta Comunidad pueda tener acceso al sistema sanitario.

En el marco del modelo conceptual de los determinantes sociales de la salud, el género se postula como uno de los ejes de desigualdad que atraviesan la estructura social y que, en interacción con otros ejes como la clase social, la edad, la etnia o la raza y el territorio, determina jerarquías de poder y conlleva desigualdades en las oportunidades de tener una buena salud (García et al., 2015).

A la hora de identificar las desigualdades de las mujeres inmigrantes, la interseccionalidad se configura como un marco de análisis que facilita profundizar en las intersecciones entre las dimensiones de género, etnia y clase social, ya que la variable género no puede ser analizada de forma aislada respecto a otras como la etnia o la clase social.

Esta línea de análisis nace de la teoría feminista y se considera como una de sus contribuciones teóricas más importantes (Cho et al., 2013).

Así se pone de manifiesto en la producción científica en varios ámbitos, entre ellos el de la salud (Bowleg, 2012; Nygre y Olofsson, 2014). 
La idoneidad de este enfoque en el abordaje de la salud de las mujeres inmigrantes se refleja en varios estudios a los que hemos tenido acceso. (Gregorio, 2017; Cardozo, 21018; Pavón, 2014).

La situación de vulnerabilidad de las mujeres inmigrantes tiene múltiples manifestaciones. Por ejemplo, en el Informe sobre Deficiencias e inequidad en los servicios de salud sexual y reproductiva en España (AIETI, 2016) se evidencia la prevalencia de la Violencia de Género entre las mujeres extranjeras duplica a la de las mujeres españolas, más del $80 \%$ de las mujeres en situación de prostitución (y/o trata), también son extranjeras y no podemos olvidarnos de la MGF en España: se estima que la población femenina en riesgo de sufrir MGF ronda las 17.000 niñas menores de 15 años.

Otro de los ámbitos donde se pone de manifiesto su vulnerabilidad es el de la Salud Sexual y Reproductiva, aspectos que trataremos en el siguiente apartado.

\section{LA SALUD REPRODUCTIVA DE LAS MUJERES INMIGRANTES}

A pesar del reconocimiento internacional sobre la importancia de la salud sexual y reproductiva (SSR), el ejercicio de los derechos humanos en este campo está fuertemente limitado y existen graves barreras que impiden a las personas - y de forma particular a las mujeres- desarrollar plenamente su sexualidad, así como acceder a servicios y suministros de salud sexual y reproductiva necesarios.

La salud y los derechos sexuales y reproductivos deben ser promovidos desde las políticas sanitarias y sociales estatales y locales e integrarse dentro del marco de la atención primaria de salud, el ámbito escolar y los servicios y recursos para jóvenes, como principales sectores (Sojo et al., 2002).

Los estudios epidemiológicos constatan que los programas de atención durante el embarazo consiguen una reducción de la mortalidad materna y perinatal, así 
como mejores resultados en el parto (Zulueta, 2011). La Organización Mundial de la Salud (OMS) indica que la mortalidad neonatal se reduce en proporción directa a la tasa de asistencia a la primera visita del control del embarazo, que debe realizarse antes de la semana doce, a un número suficiente de controles y al hecho de recibir una atención adecuada durante el parto (OMS, 2003). Una de las medidas que actualmente está considerada como la mejor de las estrategias preventivas para conseguir una reducción de la morbi-mortalidad neonatal es la atención prenatal sistematizada y programada.

Son varios los estudios que corroboran que los comportamientos reproductivos de las mujeres inmigrantes (incluyendo el uso y la demanda del sistema de salud) están condicionados tanto por los aspectos culturales y las características sanitarias de sus países de origen como por las existentes en el país de destino (Kuster et al., 2002; Luque y Oliver, 2005).

Es importante señalar que hay interés y preocupación respecto a la salud obstétrica de la población inmigrante, ya que numerosas investigaciones ponen de manifiesto que la salud reproductiva es mucho peor que la de las mujeres autóctonas.

La mayoría de las investigaciones se han llevado a cabo en países con tradición migratoria más larga. En ellas se muestran diferencias significativas entre mujeres españolas y mujeres extranjeras en lo que respecta a diferentes aspectos relacionados con la salud reproductiva, tales como una menor asistencia a los centros sanitarios para el seguimiento del embarazo en mujeres, incluso en aquellas con patologías previas al embarazo (Hussain et al., 2001; Heaman et al., 2007; El Reda et al., 2007; Tariq et al., 2012; Floridia et al., 2014), una peor percepción de la atención recibida ( Tandon et al., 2005; Dias et al., 2010) y un resultado obstétrico desfavorable (El-Sayed y Galea, 2011; Almeida et al., 2014). Los escasos estudios en el plano nacional, reflejan la existencia de una serie de desigualdades con respecto a las mujeres españolas en los aspectos relacionados con el acceso y uso del sistema sanitario (García et al., 2008), y en concreto diferencias en el proceso de atención a la salud reproductiva: embarazo, parto y puerperio (Liberal et al., 2012). 
En estos estudios, se coincide en afirmar que las mujeres de nacionalidades diferentes a la española presentan una frecuencia significativamente mayor de tener un embarazo con un control inadecuado, sobre todo las marroquíes (Manzanares et al., 2008).

La idoneidad de la incorporación del enfoque interseccional en el estudio de las desigualdades en la salud reproductiva de las mujeres inmigrantes cada vez es más compartida. Así se evidencia en los estudios recientes a los que hemos tenido acceso (Cardozo, 2014; Ramírez, 2017).

\subsection{Principales barreras de acceso y uso de la atención perinatal}

Entre los factores que contribuyen a la situación de vulnerabilidad de las mujeres inmigrantes, en cuanto a que dificultan el acceso y uso de la atención perinatal, destacamos las propias características del contexto migratorio, el abordaje biomédico de la atención a la salud y la escasa competencia cultural del sistema sanitario.

\section{Características del contexto migratorio}

En el caso de las mujeres inmigrantes, los estudios a los que hemos tenido acceso concluyen que las recién llegadas a su país de acogida, con escasos apoyos sociales, dificultades idiomáticas y limitados conocimientos sobre el sistema sanitario tienen un mayor riesgo de recibir una atención perinatal no adecuada (Sanmartin y Ross, 2006; Hayes et al., 2011; Boerleider et al., 2013; Ugarte, 2015).

En este sentido, la denominada "ceguera de género en la investigación social" (Pavón, 2014:101), ha invisibilidad las características diferenciales entre hombres y mujeres en el proceso migratorio. Sólo desde un enfoque interseccional de género, etnia y clase social podemos llegar a identificar las desigualdades y diseñar propuestas de cambio. 
Respecto a la influencia de los factores culturales, podemos decir que los comportamientos reproductivos de las mujeres inmigrantes (incluyendo el uso y la demanda del sistema de salud) están condicionados tanto por los aspectos culturales y las características sanitarias de sus países de origen como por las existentes en el país de destino (Kaplan, 2001;Kuster et al. 2002; Dias et al., 2010).

\section{Abordaje biomédico de la atención a la salud}

Bajo el paradigma biopsicosocial para el abordaje de los conceptos salud y enfermedad, se supera el bioligicismo que durante tanto tiempo impregnó la medicina. La atención a la salud de las personas se realiza de manera integral, reconociéndose la incorporación de las dimensiones psicológicas, sociales y culturales como determinantes de la salud y de necesario reconocimiento en los encuentros asistenciales. Sin embargo, a pesar de que en el plano teórico queda claro y haya un consenso más que justificado en la incorporación de este enfoque integral, en el plano operativo, en el día a día de la atención sanitaria, las dificultades son un hecho. Es decir que, aunque se promueven los principios y estrategias de la atención biopsicosocial a nivel legislativo y en los programas de atención, en la práctica diaria prevalece el modelo de atención biologicista (Ugarte, 2012).

La relación asistencial se desarrolla en el marco de un modelo que, aunque en la teoría contempla las dimensiones socioculturales de la salud y la enfermedad en sus procesos de atención, en la realidad se evidencia la influencia de sesgos biomédico, etnocéntrico y culturalista en el abordaje de estas dimensiones. Así, la mirada de los y las profesionales de la salud no es neutra, está tamizada por estos sesgos que condicionan la propia mirada, lo que se ve, la interpretación de lo que se ve y lo que se hace, las prácticas que se materializan en el encuentro.

El sesgo biomédico conlleva que el modelo de atención se realice a través de protocolos estandarizados que permiten llevar a cabo un tratamiento homogéneo de atención a las dimensiones biológicas, dejando al margen las dimensiones psicológica, social y cultural. 
Además, la visión positivista del actual modelo hegemónico en la atención a la salud-enfermedad, en la que "la definición medica de la enfermedad como una desviación de una norma biológica; la doctrina de que existe una etiología específica de las enfermedades; la noción de que las enfermedades son universales; la idea de que la medicina es neutral; o la dicotomía mente/cuerpo" (Esteban, 2006: 12), está vinculada a la resistencia a aplicar la categoría de género que aporta la perspectiva feminista. El androcentrismo del sistema patriarcal tiene su impronta en el modelo actual de la atención sanitaria, en el que se evidencia el sesgo de género, entendido como «el planteamiento erróneo de igualdad o de diferencias entre hombres y mujeres -en su naturaleza, sus comportamientos y/o sus razonamientos-, el cual puede generar una conducta desigual en los servicios sanitarios (incluida la investigación) y es discriminatoria para un sexo respecto al otro» (Ruiz-Cantero y Verdú-Delgado, 2004:119).

El sesgo etnocéntrico conlleva que el modelo de atención no pueda responder a un protocolo estandarizado de atención a las dimensiones socioculturales. Este sesgo ocasiona que la observación y el tratamiento de las diferencias se aborde de forma etnicista, etnocéntrica, descontextualizada y monocultural. Es decir, aun advirtiendo que las poblaciones inmigrantes son diferentes socioculturalmente la tendencia practicada promueve prestar una atención homogénea ante ellas (Gijón, 2014).

Y por último, el sesgo culturalista, definido como la exageración de los factores culturales y la esencialización de la cultura. Las consecuencias de este sesgo es que se interpretan los comportamientos de las personas por su pertenencia a una determinada cultura (Ugarte, 2015).

Consideramos necesario aclarar que si bien es cierto que las relaciones asimétricas y de subordinación (poder de los y las profesionales de la salud) características del modelo de atención sanitaria con un enfoque biomédico predominante en el sistema sanitario español, propicia que una persona usuaria de dicho sistema se sienta en "territorio extraño" aun siendo autóctona, la situación de las personas inmigrantes es aún más grave (Triviño, 2012; Juárez y Saforcada, 2014). Así, la relación entre ambas partes se construye desde la 
desigualdad. Desde una perspectiva feminista, podríamos decir que el actual modelo de atención sanitaria es un "producto" del sistema patriarcal, construido con sus principios y valores y en el que las relaciones de poder están bien demarcadas. El funcionamiento del modelo de atención a la salud es un reflejo del modelo patriarcal. En él se reproduce el sistema de relaciones de poder, tanto en el plano institucional como en la práctica de la atención, ya que los y las profesionales de la salud están socializadas y reciben la formación en un contexto patriarcal.

\section{Deficiente competencia cultural del sistema sanitario}

Otro de los factores que se ha visto que dificulta el acceso y uso de los servicios sanitarios es la deficiente competencia cultural de los mismos, tanto a nivel estructural como a nivel profesional. En un contexto donde la diversidad cultural es una realidad constante los y las profesionales de la salud tienen que ser capaces de manejarse y dar respuestas satisfactorias a las necesidades de pacientes de diferentes culturas.

Por este motivo es necesario el desarrollo de unos modelos de salud que respondan a las necesidades de las personas usuarias del sistema de salud, planteándose la interculturalidad como un proceso de acercamiento entre los distintos sistemas de atención sanitaria (Alarcón et al., 2003).

Así lo dice la OMS (2001) en uno de sus documentos sobre salud e inmigración, donde señala que un elemento fundamental del derecho a la salud es que todos los establecimientos, bienes y servicios de salud deben estar adaptados a esa diversidad cultural como modo de evitar errores diagnósticos y mejorar la accesibilidad.

Se define el concepto de competencia cultural en los sistemas sanitarios, como la capacidad del sistema sanitario y sus profesionales para valorar los factores socioculturales de los y las pacientes, como una parte más de sus competencias profesionales, operativizando de esta manera el enfoque biopsicosocial de la atención a la salud. Es la adaptación de la atención sanitaria de manera 
congruente con la cultura del o de la paciente. Esta competencia es un proceso dinámico, mediante el cual el o la profesional de la salud o un sistema sanitario busca proporcionar el cuidado a través de estrategias basadas en el conocimiento del patrimonio cultural, en las actitudes y en los comportamientos de aquellas personas a quienes dirige sus cuidados (Giger et al., 2007).

La competencia cultural se fundamenta en dos principios básicos: primero, es importante explorar y comprender los factores socioculturales que influyen en los valores, las creencias y los comportamientos de las personas relacionados con la salud y la asistencia sanitaria; y segundo, es fundamental para el desarrollo de estrategias intersectoriales en el diseño y la prestación de la asistencia sanitaria en un esfuerzo por reducir las brechas en la calidad que resultan de las barreras lingüísticas y socioculturales.

Con estas características, el sistema sería equitativo, de alta calidad, y libre de las disparidades basadas en las características individuales del o de la paciente. En definitiva, lo que se pretende es facilitar el proceso para que los cuidados que se oferten sean congruentes con el contexto cultural de la persona usuaria de los servicios sanitario (Bridge et al., 2008).

Cabe señalar que la eficacia de los programas que aplican la competencia cultural en la atención a pacientes inmigrantes está ampliamente documentada (Ugarte, 2015).

Las dimensiones de esta competencia son la sensibilidad cultural, que hace referencia a las habilidades interpersonales y al establecimiento de unas relaciones significativas mediante el logro de la confianza entre las dos partes del encuentro (Taylor et al., 2011): la habilidad cultural, que se traduce en la capacidad de recabar información culturalmente relevante para abordar un determinado problema de salud y realizar una valoración precisa (Leininger, 2001) conocimiento cultural (Foronda, 2008) y la conciencia cultural, relacionada con el autoexamen y el reconocimiento de los prejuicios (Belintxon y De Castillo, 2014). 
Una buena comprensión de la competencia cultural pasa por considerarla como una estrategia de abordaje de la atención a la salud en los contextos de diversidad cultural y partiendo de la premisa de la heterogeneidad en los grupos y entendida como un proceso.

La pertinencia de la competencia cultural de los y las profesionales a la hora de promocionar el acceso al sistema sanitario en los contextos de diversidad cultural está avalada por numerosos estudios que lo refrendan e incluso se llega a valorar como más eficiente que el uso de otras herramientas como los traductores telefónicos o los y las intérpretes (Ugarte, 2015).

En la revisión de la literatura científica constatamos que cada vez hay un mayor interés en abordar conjuntamente la perspectiva intercultural y la perspectiva de género. Hacer esta sinergia facilita conocer las causas profundas de la desigualdad y sus intersecciones. Se plantea un análisis interseccional que incardine el enfoque de derechos humanos, el enfoque de género y el de la interculturalidad (UNFPA et al,2012).

\section{POSIBLES LÍNEAS DE ACCIÓN}

A continuación, se presentan algunas de las posibles líneas de acción con el objetivo de disminuir la inequidad en salud de las mujeres inmigrantes en su proceso perinatal, extraídas de nuestra investigación del proyecto de Tesis Doctoral (Ugarte, 2015) y del análisis de otros estudios relacionados con la temática del nuestro.

Creemos necesario incorporar el enfoque de la interseccionalidad como herramienta clave a la hora analizar con una mayor profundidad las desigualdades en salud de las personas inmigrantes, concretamente las que sufren las mujeres en los procesos migratorios. 
Sin duda, el abordaje estratégico debe ser integral, implementándose medidas en tres niveles, configurando una serie de acciones que previamente deberán pasar por un análisis de su viabilidad.

Entre las acciones dirigidas a profesionales de la salud, se podrían valorar la formación en la competencia cultural, en todas sus dimensiones: conciencia, sensibilidad, conocimiento y habilidades culturales; la inclusión en los planes de estudios de Grado, Postgrado y en la formación continuada de profesionales de la salud disciplinas encaminadas a fomentar las competencias para abordar en condiciones de equidad las desigualdades sociales en salud y la diversidad cultural a partir de la Teoría Feminista y la información a profesionales de la salud sobre el derecho de las mujeres a escoger los y las profesionales y garantizar su cumplimiento.

Respecto a las acciones dirigidas a las mujeres, se podrían analizar las posibles alternativas dirigidas hacia la alfabetización de las mujeres, entendiendo la alfabetización como la adquisición de la competencia lingüística en castellano; el conocimiento de la estructura, organización y oferta asistencial del sistema sanitario y sus prestaciones; el conocimiento de los recursos sociosanitarios del ámbito geográfico de su residencia (transporte, ubicación de centros sociales y sanitarios) y el conocimiento de sus derechos y obligaciones como usuarias de los servicios de salud.

Por otra parte, ya en el plano institucional, la exploración de posibles alternativas de funcionamiento iría encaminada a valorar aspectos como la incorporación explícita de los enfoques biológicos, psicológicos, sociales y culturales en el modelo de atención a la salud, reconociendo la heterogeneidad de las mujeres más allá de las diferencias culturales. 
Por otra parte, se hace necesario un enfoque interseccional en las políticas sanitarias desde el que se diseñen estrategias de intervención que aborden a las mujeres inmigrantes no como un colectivo único y homogéneo y que permita el análisis de en términos de género, etnia y clase social.

\section{BIBLIOGRAFÍA}

Alarcón, Ana María; Vidal, Aldo y Neira, Jaime (2003). Salud intercultural: elementos para la construcción de sus bases conceptuales. Revista Médica de Chile, 131(9), 1061-1065.

Almeida, Joana; Mulready-Ward, Candace; Bettegowda, Vani y Ahluwalia, Indu (2014). Racial/ethnic and nativity differences in birth outcomes among mothers in New York City: the role of social ties and social support. Maternal \& Child Health Journal,18(1), 90-100.

Belintxon, Maider y de Dicastillo, Olga (2014). Los retos de la promoción de la salud en una sociedad multicultural: revisión narrativa de la literatura. Paper presented at the Anales del Sistema Sanitario de Navarra.

Boerleider, Agatah ; Wiegers, Therese; Manniën, Judith; Francke, Anneke y Devillé, Walter (2013). Factors affecting the use of prenatal care by nonwestern women in industrialized western countries: a systematic review. BioMed Central Pregnancy \& Childbirth, 13, 81-81. doi:10.1186/1471-2393-13-81

Bowleg, Lisa (2012). El problema con la frase mujeres y minorías: interseccionalidad, un importante marco teórico para la salud pública. Revista estadounidense de salud pública, 102 (7), 1267-1273. doi: 10.2105 AJPH.2012.300750

Bridge, Tana J; Massie, Enos Greer y Mills, Crystal (2008). Prioritizing cultural competence in the implementation of an evidence-based practice model. Children and Youth Services Review, 30(10), 1111-1118 doi:http: / / dx.doi.org/10.1016/j.childyouth.2008.02.005

Cardozo, Sofía (2018). Mujeres que migran: atención en salud sexual y reproductiva a migrantes afrocaribeñas en Uruguay. ENCUENTROS LATINOAMERICANOS (Segunda ÉPoca) ENCLAT ISSN 1688-437X, 2(2), 49-85 Consultado de: http: / / ojs.fhuce.edu.uy/index.php/enclat/article/view / 124

Cho, Sumi; Crenshaw, Kimberlé Williams; McCall, Leslie (2013). Toward a field of Intersectionality Studies: theory, applications and praxis. Signs: Journal of Women in Culture and Society, 38(4), 785-810. 
Dias, Sonia; Gama, Ana y Rocha, Critianne (2010). Immigrant women's perceptions and experiences of health care services: Insights from a focus group study. Journal of Public Health, 18(5), 489-496.

El Reda, Darline; Grigorescu, Violanda; Posner, Samuel y Davis-Harrier, Amanda (2007). Lower rates of preterm birth in women of Arab ancestry: an epidemiologic paradox--Michigan, 1993-2002. Matern Child Health J, 11(6), 622-627. doi:10.1007/s10995-007-0199-y

El-Sayed, Abdulrahman y Galea, Sandro (2011). Interethnic mating and risk for preterm birth among Arab-American mothers: Evidence from the Arab-American Birth Outcomes Study. Journal of immigrant and minority health / Center for Minority Public Health, 13(3), 445-452. doi:10.1007/s10903-010-9341-9

Esteban, Mari Luz (2006): El estudio de la salud y el género: Las ventajas de un enfoque antropológico y feminista. Salud Colectiva, 2 (1), 9-20.

Floridia, Marco; Pinnetti, Carmela; Ravizza, M; Frisina, V; Cetin, I y Fiscon, Marta (2014). The Italian Group on Surveillance on Antiretroviral Treatment in, P. Rate, Predictors, and Consequences of Late Antenatal Booking in a National Cohort Study of Pregnant Women With HIV in Italy. HIV Clinical Trials, 15(3), 104-115. doi:10.1310/hct1503-104

Foronda, Cynthia (2008). A concept analysis of cultural sensitivity. Journal Transcultal Nursing, 19(3), 207-212. doi:10.1177/1043659608317093

García, Juan; Pardo, Carmen; Hernández, Antonio; Lorenzo, Marca y Gil, Diana (2008). Diferencias obstétricas y neonatales entre mujeres autóctonas e inmigrantes. TITLEREVISTA, 51(02), 53-62

García, María del Mar; Del Río, María y Marcos, Jorge (2015). Guía de indicadores para medir las desigualdades de género en salud y sus determinantes. Escuela Andaluza de Salud Pública. Consejería de Sanidad. Junta de Andalucía. Consultado de: https: / / www.easp.es/ project/ guia-de-indicadores-para-medir-lasdesigualdades-de-genero-en-salud-y-sus-determinantes /

García, Yedra; Aguilera, Eva; Reyero, Miguel y Martín, Sagrario (2016). Deficiencias e inequidad en los servicios de salud sexual y reproductiva en España. Editan: Alianza por la Solidaridad Asociación de Investigación y Especialización sobre Temas Iberoamericanos (AIETI) et al.

Giger, Joyce; Davidhizar, Ruth E; Purnell, Larry; Harden, J Taylor; Phillips, Janice y Strickland, Ora (2007). American Academy of Nursing Expert Panel report: developing cultural competence to eliminate health disparities in ethnic minorities and other vulnerable populations. Journal Transcultal Nursing, (18), 95-102.

Gijón, María Teresa (2014). El espejismo de lo sociocultural en salud: discursos socio-profesionales sobre el encuentro asistencial con poblaciones 
inmigrantes desde el Modelo de Atención Primaria al Sur de Europa. Granada: Universidad de Granada.

Gregorio Gil, Carmen (2017). Etnografiar las migraciones 'Sur'- 'Norte': la inscripción en nuestros cuerpos de representaciones de género, raza y nación. Empiria. Revista de metodología de ciencias sociales, 0(37), 1939. doi:10.5944/empiria.37.2017.18975

Hayes, Jon; Enohumah, K. yMcCaul, C (2011). Care of the migrant obstetric population. International Journal of Obstetric Anesthesia, 20(4), 321-329. doi:10.1016/j.ijoa.2011.06.008

Heaman, Maureen ; Green, Chris ; Newburn-Cook, Christine; Elliott, Lawrence y Helewa, Michael (2007). Social inequalities in use of prenatal care in Manitoba. Journal Obstetrics Gynaecology Canada, 29(10), 806-816.

Hussain, Akhtar; Moodley, Dhayendre; Naidoo, Sunhindra y Esterhuizen, Tonya (2001). Pregnant Women's Access to PMTCT and ART Services in South Africa and Implications for Universal Antiretroviral Treatment. PLoS ONE, 6(12), e27907. doi:10.1371/journal.pone.0027907

Kaplan, Adriana (2001). Integración social, identidad étnica y género: la salud sexual y reproductiva de las mujeres migrantes subsaharianas: retos e implicaciones. Paper presented at the Comunicación del II Congreso sobre la inmigración en España.

Juárez, María Paula y Saforcada, Enrique (2014). El problema de la barrera psicosociocultural interpuesta entre los profesionales de la salud y consultantes de contextos pobres estructurales. Reflexiones sobre una cuestión compleja [The problem of psycho-socio barrier interposed]. Salud y Sociedad, 4(3), 210-227.

Kuster, Marianne; Goulet, Céline y Pépin, Jacinth (2002). Significations du soin Postnatal pour des immigrants algériens. L'infirmière du Quebec, 10(1), 12-23.

Leininger, Madeleine (2001). Culture care diversity and universality: A theory of nursing. New York: National League for Nursing Press.

Liberal, Marisa; Garrido, Nuria; Tello, Enric; Mestanza, JA y Iglesias, Enrique (2012). Estudio de las diferencias obstétricas y perinatales entre gestantes españolas e inmigrantes en nuestro área de salud Study of obstetrics and perinatal differences between Spanish and foreign immigrant women in our health area. PRÁCTICA, 134-140.

Luque, Miguel Ángel y Oliver, María Isabel (2005). Diferencias culturales en la percepción y vivencia del parto: El caso de las mujeres inmigradas. Index de Enfermería, (48-49), 9-13.

Manzanares, Sebastián; López, María Felicidad; Gómez, Teresa; Martínez, Nina y Montoya, Francisco (2008). Resultados del mal control del embarazo en la población inmigrante. TITLEREVISTA, 51(04), 215-223. 
Ministerio de Sanidad, Servicios Sociales e Igualdad. Comisión para reducir las desigualdades sociales en salud en España (2015). Avanzando hacia la equidad. Madrid: Ministerio de Sanidad, Servicios Sociales e Igualdad.

Nygren, Katarina G y Olofsson, Anna (2014). Intersectional approaches in health-risk research: a critical review. Sociology Compass, 8(9), 11121126. doi: $10.1111 /$ soc4.12176.

OMS. (2001). National standards for culturally and linguistically appropriate services in health care. Final report, U.S. Department of Health and Human Services, Rockville, Offi ce of Minority Health.

OMS. (2003). International migration, health and human rights. (Vol. 4). Health and Human rights publication series: Ginebra: OMS.

Pavón, Estela (2014). Feminismo, Género e Inmigración. En Chocarro, Edurne y Sáenz, $\mathrm{M}^{\mathrm{a}}$ del Carmen (coord.), Oriente y Occidente: la construcción de la subjetividad femenina (pp.101-118). Logroño: Universidad de La Rioja.

Ramírez, Dulce Karol (2017). Mujeres migrantes en la frontera sur de México: aproximaciones desde la interseccionalidad. Tuxtla Gutiérrez, Chiapas: CESMECA-UNICACH.

Ruiz-Cantero, María Teresa y Verdú-Delgado, María (2004). Sesgo de género en el esfuerzo terapéutico. Gaceta Sanitaria, 18(Supl. 1), 118-125. Consultado

en: http: / / scielo.isciii.es / scielo.php?script=sci_arttext\&pid=S021391112004000400019\&lng=es\&tlng=es

Sanmartin, Claudia y Ross, Nancy (2006). Experiencing difficulties accessing first-contact health services in Canada: Canadians without regular doctors and recent immigrants have difficulties accessing first-contact healthcare services. Reports of difficulties in accessing care vary by age, sex and region. Healthcare Policy, 1(2), 103.

Sojo, Diana; Sierra, Irene y López, Irene (2002). SALUD Y GÉNERO. Guía práctica para profesionales de la cooperación. Madrid: Médicos del Mundo.

Tandon, S Darius; Parillo, Kathleen y Keefer, Maureen (2005). Hispanic women's perceptions of patient-centeredness during prenatal care: a mixed-method study. Birth, (32), 312-317.

Tariq, Sema; Pillen, Alex.;Tookey, Pat; Brown, Alison y Elford, Jonathan (2012). The impact of African ethnicity and migration on pregnancy in women living with HIV in the UK: design and methods. BioMed Central Public Health, 12, 596-596. doi:10.1186/1471-2458-12-596

Taylor, Georgina; Papadopoulos, Irena; Dudau, Victor; Maerten, Michael; Peltegova, A y Ziegler, M (2011). Intercultural education of nurses and health professionals in Europe (IENE). International Nursing Reviev, 58(2), 188-195. doi:10.1111/j.1466-7657.2011.00892.x 
Triviño, Rosana (2012). Mujeres migrantes y misoprostol: aborto privado, escándalo público. Dilemata, (10), 31-44.

Ugarte, María Idoia (2012). La evidencia narrativa y la calidad asistencial en el Sistema Nacional de Salud. En Lorenzo, Mariano y Cipriano, Carmen (Eds.) Medicina y Narrativas. De la teoría a la práctica, (pp. 95-118). Madrid: Círculo Rojo.

Ugarte, María Idoia (2015). La salud reproductiva de las mujeres inmigrantes marroquíes. Análisis cualitativo de las representaciones sociales de mujeres y profesionales de la salud. [Tesis Doctoral en Internet]. [Albacete]: Universidad de Castilla-La Mancha. Consltado en: http: / / hdl.handle.net/10578/9051

UNFPA, ONU Mujeres, UNICEF y PNUD (2012): Ampliando la mirada. La integración de los enfoques de género, interculturalidad y derechos humanos. Santiago de Chile.

Zulueta, Mari Paz (2011). Efectividad de una intervención socio-sanitaria dirigida a disminuir las desigualdades en salud reproductiva de las mujeres inmigrantes marroquíes en Cantabria. REDUCA (Enfermería, Fisioterapia y Podología), 3(3).

Xu, Ke; Evans, David; Carrin, Guido; Aguilar-Rivera, Ana Mylena; Musgrovey, Philip y Evans, Timothy (2007). Protecting households from catastrophic health spending. Health Affairs, 26, 972-983. 